\title{
Perception des institutions de sécurité et de justice par les abidjanais
}

\section{The Abidjanian perception of security and justice institutions}

\author{
Antoine Nassoua Okpo
}

\section{Riassunto}

L'obiettivo di questo studio è quello di analizzare la percezione e la rappresentazione indotta delle istituzioni di sicurezza e giustizia ivoriane da parte degli abitanti di Abidjan. Le teorie della motivazione, in particolare quelle del contenuto motivazionale, sono state utilizzate per comprendere i giudizi espressi dagli abitanti di Abidjan. Un questionario e alcune interviste hanno consentito di raccogliere dati da cui emerge un'immagine negativa del sistema di sicurezza e di giustizia ivoriano, immagine che si è progressivamente sedimentata nella coscienza collettiva della popolazione. Questa svalutazione del sistema è motivata dalla differenti esperienze di insoddisfazione e da altre doglianze nei confronti del sistema e dei suoi operatori.

\section{Résumé}

Cette étude vise à connaittre la perception et la représentation induite des institutions de sécurité et de justice ivoiriennes par les abidjanais. Les théories de la motivation, notamment celle du contenu motivationnel ont été utilisées pour comprendre le jugement des abidjanais. Un questionnaire et des entretiens ont permis de recueillir des données dont l'analyse relève une image négative du système de sécurité et de justice ivoirien qui s'est progressivement construite dans la conscience de la population. Cette dépréciation du système est motivée par les différentes expériences d'insatisfaction et autres griefs à l'endroit du système et de ses agents.

\section{Abstract}

This study aims at knowing the Abidjanian perception and the induced representation of Ivorian institutions of security and justice. We used theories of motivation, in particular the one concerning motivational content, in order to understand the Abidjanian judgments. Data from a questionnaire and some interviews have provided information that highlight a negative image among citizens of the Ivorian system of security and justice. This discrediting of the system is mainly the result of different dissatisfaction experiences and other complaints towards the system and its agents.

Key words: insecurity perception; Ivorian institutions of security and justice; theories of motivation; dissatisfaction experiences.

\section{Introduction : quelques repères théoriques. \\ Les écrits portent très souvent sur les expériences vécues dans les institutions de sécurité et de justice et la construction de} l'identité individuelle ou collective.

\footnotetext{
• Maître-Assistant, UFR Criminologie, Université Félix Houphouët Boigny de Cocody Abidjan, Côte d'Ivoire.
} 
Selon Chaussebourg ${ }^{1}$, l'identification et la condamnation de l'agresseur, la protection des victimes et l'empêchement de la reproduction de l'incident sont les attentes légitimes des victimes. Aussi, Lachambre ${ }^{2}$ indique que la volonté de dénoncer le crime est liée à l'attente du public. Cette attente est l'imposition d'une peine proportionnelle au niveau de la désapprobation du public à l'égard du crime. Si ces objectifs ne sont pas atteints, les plaignants et les victimes ne voient pas l'utilité de la dénonciation. Ces résultats sont également indiqués par Sidebottom $^{3}$ pour qui de nombreuses victimes de criminalité n'informent pas la police. Et que cette décision de déclarer les violences subies est liée aux facteurs personnels de la victime, à l'événement de la criminalité et de la communauté plus large. Perreault et Brennan ${ }^{4}$ relèvent plusieurs raisons pour lesquelles une victime peut décider de signaler ou de ne pas signaler un incident à la police. Deux raisons sont beaucoup plus déterminantes selon les personnes interrogées. L'une tient au sens du devoir du citoyen et l'autre pour faire arrêter et punir l'auteur. Algava et Bèque ${ }^{5}$ avaient

1 Chaussebourg L., Se déclarer victime: de l'atteinte subie au dépôt de plainte, Infostat Justice, avril 2010, $n^{\circ} 109$, Ministère de la justice et des libertés, France.

${ }^{2}$ Lachambre S., La théorie de la dénonciation: émergence et institutionnalisation en droit criminel, Thèse soumise à la faculté des études supérieures et postdoctorales dans le cadre des exigences du programme de doctorat en criminologie. Département de criminologie, Faculté des sciences sociales, Université d'Ottawa, 2011.

${ }^{3}$ Sidebottom A., «On the correlates of Reporting Assault to the Police in Malawi », The British Journal of Criminology, vol. 55, n. 2, 2015.

${ }^{4}$ Perreault S., Brennan S., La victimisation au Canada, 2009, Centre Canadien de la statistique juridique, Satatistics Canada-N85-002X, 2010.

${ }_{5}$ Algava E., Bèque M., «Perception et vécu des comportements intolérants. Une analyse du modèle également souligné une double dimension de la plainte. Car la référence à Fassinn ${ }^{6}$ quant à la souffrance générée par les attitudes ou comportements de discrimination, les amène à affirmer que la «plainte comporte, historiquement et sémantiquement une double dimension: l'une juridique, qui est l'exigence d'un droit ("porter plainte"), l'autre affective, qui est l'expression d'une souffrance ». Ces auteurs voient dans la plainte l'expression d'une souffrance qui déborde largement la dimension judiciaire. C'est pourquoi, elles indiquent que la déclaration d'un incident à la police tient davantage à des raisons propres au type de crime, puisque les victimes de crimes violents étaient plus susceptibles que les victimes de crimes contre les ménages de signaler l'incident à la police. Les premières réclamant la protection alors que les secondes souhaitant bénéficier de l'assurance ou d'une indemnité.

Si assurer la sécurité et la protection des biens et des personnes par les autorités de police et judiciaires est purement formel et théorique, quelle est l'image que les abidjanais retiennent-ils de ces institutions? Quels sont les comportements induits par la perception, la représentation subjective des abidjanais en ce qui concerne ces institutions?

Selon Perreault et Brennan ${ }^{7}$, les canadiens sont généralement satisfaits de leur sécurité personnelle, tout comme le mentionnent

\footnotetext{
"Relation aux autres', de l'enquête Histoire de vie », Economie et statistique, volume $393, \mathrm{n}^{\circ} 1$, 2006.

${ }^{6}$ Fassin D., The embodiment of inequality, 2003, Document produit en version numérique, site web : http://www.ncbi.nlm.nih.gov/pmc/articles/PMC132 6443/

${ }^{7}$ Perreault S., Brennan S., op. cit.
} 
également Killias et al. ${ }^{8}$; les suisses interrogés dans le cadre de leur étude sont globalement satisfaits du travail de la police. Et pour cette raison, la volonté de la victime à rapporter les infractions est moins influencée par l'image de la police. Cette précision est de taille puisque ces personnes victimes sont déjà satisfaites du travail de la police. Or, dans le cas de l'étude d'Abidjan, bien que l'échantillon ne soit pas représentatif, la population semble ne pas être satisfaite du travail des institutions de sécurité et de justice. Les abidjanais adoptent une solution de résignation quant au règlement de leur litige. L'opinion générale des victimes sur le système de justice résultant de leur insatisfaction entraîne selon elles, un sentiment d'insécurité générale et la crainte de leur sécurité personnelle. Ainsi, l'insatisfaction explique-t-elle la réticence quant au signalement de l'infraction subie par la grande majorité des victimes d'actes criminels à Abidjan? Si tel est le cas, quelle est l'image des institutions étatiques de sécurité et de justice retenue par la population abidjanaise? Une première réponse est obtenue avec $\mathrm{Hill}^{9}$ qui recherchait les conséquences psychologiques du processus de traumatisme à la police. Selon lui, « en agissant convenablement, le personnel du système de justice pénale peut avoir une influence positive sur les victimes. Car, pour les victimes, l'enquête et l'arrestation de l'infracteur sont des éléments positifs qui accroissent la

\footnotetext{
${ }^{8}$ Killias M., Staubli S., Biberstein L., Bänziga M., Iadanza S., Sondage au sujet des expériences et opinions sur la criminalité en Suisse. Analyse dans le cadre sondage national de victimisation 2011, Institut de criminologie, Université Zürich, 2011.

${ }^{9}$ Hill K. J., Réaction des victimes au traumatisme et conséquences sur les interventions: étude et synthèse de la documentation, Ministère de la Justice, Canada, 2003.
}

perception que la police a été utile ». La référence faite à Norris et coll. amène Hill à conclure que «le fait de considérer que la police avait été utile réduisait l'aliénation de la victime ». Aucoin et Beauchamp ${ }^{10}$ rapportent que la perception du système de justice par les victimes est fonction de leur expérience avec le système judiciaire, car les victimes peuvent avoir des opinions différentes du système judiciaire que les non victimes. Et que la confiance et la perception de la victime à l'égard de l'efficacité du processus judiciaire peuvent être affectées en fonction de l'expérience de la victime. Ainsi, soutiennent-elles que la perception par les victimes de leur quartier, de leur sécurité personnelle de même que du système policier est affectée par leur expérience de la victimisation antérieure. Au total, le fait d'être victime d'un crime semble avoir un impact négatif sur la perception qu'a une personne de la police et de son efficacité.

Ainsi, la perception du système de justice est fonction de l'agissement du personnel du système et du système lui-même. La perception est dans ce cas déterminante pour appréhender les agissements des agents et du système en général. Comment la définir et que retenir pour l'évaluation du travail du système en général? Selon Sillamy ${ }^{11}$, «la perception est une conduite psychologique complexe par laquelle un individu organise ses sensations et prend connaissance du réel. - La perception est un rapport du sujet à

\footnotetext{
10 Aucoin K., Beauchamp D., Répercussion et conséquences de la victimisation, ESG 2004. Statistique Canada-n ${ }^{\circ}$ 85-002-XIF au catalogue, vol. 27, $\mathrm{n}^{\circ} 1,2008$, Ottawa, Centre Canadien de Statistique Juridique.

${ }^{11}$ Sillamy N., Dictionnaire de la psychologie, Larousse, Evreux, 1976.
} 
l'objet: celui-ci a ses caractéristiques propres, mais c'est avec ma subjectivité que je le perçois ; dans ma façon de l'appréhender se projettent ma manière d'être, ma façon de penser, modérée par mes expériences antérieures et le milieu socioculturel auquel j’appartiens, et mes intérêts immédiats ». Dans cette perspective, le recours à Gimenez $^{12}$ apporte un éclairage quant à la perception qu'il considère comme la construction d'une signification. C'est-à-dire la représentation ou l'image de la population est en relation avec un fond qui fait référence à un idéal à atteindre et l'appréhension de cette substance; le travail des institutions de justice, est fonction de la croyance de cette population. Puisque selon Tremblay ${ }^{13}$, les institutions sont la forme concrète, immédiate, quotidienne de la vie sociale. Il en découle une image générale qui façonne la manière de voir, de penser et de se comporter de la population.

Selon Bansept ${ }^{14}$, une image de la police nationale française reste bonne, mais en déclin. Les appréciations concernant la police émises par les citoyens (population) sont très variées et mitigées. Ainsi, il existe une différence entre la bonne opinion des valeurs de la police et de sa conduite, entre la satisfaction de son activité et de sa capacité à intervenir rapidement. Les chercheurs, comme le public français, attestent que l'action de la police est efficace lorsque des

\footnotetext{
${ }^{12}$ Gimenez M., La psychologie de la perception, Flamarion, 1997.

${ }^{13}$ Tremblay R., Vers une écologie humaine, 1990. Document produit en version numérique, site web : http://classiques.uqac.ca/

14 Bansept C., Prévention de la délinquance, mobilisation civile et recherche de la juste peine. Sécurité publique et relations police-population: passer d'une police d'ordre à une police au service du citoyen. Rapport de mission du conseil national
}

vies sont en danger. Néanmoins, les perceptions diffèrent en ce qui concerne la lutte quotidienne contre la délinquance. Les femmes particulièrement observent un laxisme dans le comportement, la manière d'être des fonctionnaires. Globalement, les populations reprochent aux agents de ne pas établir assez de contacts avec les citoyens. Un sentiment d'insatisfaction et de peur domine au sein de la population des quartiers sensibles. La police française est davantage perçue comme une force punitive plutôt que comme une force protectrice. Ce constat fait que les contextes locaux sont d'autant plus tendus entre les populations et les policiers que l'effort de prévention est faible, les liens inexistants, exclusivement limités aux contrôles d’identité. Dans cette perspective, il est fort probable qu'il existe une relation entre les agissements des agents de la police en particulier et des institutions en général, et l'image post-perceptive retenue de celles-ci par la population.

Algava et Bèque ${ }^{15}$ indiquent une part importante, au moins subjectivement, des assignations subies dans la construction de l'identité individuelle. Elles montrent à travers leur étude que les personnes ayant rapporté au moins un comportement négatif considèrent que cela a eu des conséquences parfois lourdes, sur leur vie, d'ordre matériel, professionnel, relationnel ou psychologique. Cependant, elles relativisent leur propos car nombreux sont ceux qui disent aussi en avoir tiré une source d'énergie supplémentaire. Cette identité construite est-elle à la base de la perception? Une réponse à cette

des villes à Montréal du 28 septembre au 3 octobre 2008, Montréal, 2013.

${ }^{15}$ Algava E., Bèque M., op. cit. 
interrogation est obtenue avec Gimenez ${ }^{16}$ qui affirme que le sujet produit avec ses structures biologiques et psychologiques, à partir des expériences dont il dispose, un ensemble d'idées, de théories, de «lois de la nature» qui constituent son monde. Il ajoute que de ce point de vue, la perception est source d'expérience, donc de connaissance sur le monde. Ainsi, nous pouvons répondre par l'affirmative que l'identité construite avec ses subjectivités et ses expériences permet d'avoir une image globale de l'environnement de l'individu. Une autre réponse à l'interrogation cherchant à connaittre le fondement de la perception peut provenir de la théorie de la motivation, notamment les théories $\mathrm{du}$ contenu motivationnel de Maslow et Herzberg ${ }^{17}$. En fait, les objectifs visés sont de connaître et de comprendre les explications de la représentation induite de la perception du système de sécurité et de justice ivoirien. Le recours à Thill ${ }^{18}$ indique une construction progressive de la motivation à la base du comportement. «La motivation est le terme utilisé pour décrire et expliquer les forces qui, chez un sportif d'exception comme chez tous les organismes (animaux ou humains), déclenchent le comportement, lui confèrent une orientation sélective, lui donnent une certaine intensité et persistance ». L'auteur veut montrer la manière dont une conduite est initiée et comment elle se développe au fil du temps. L'initiation et le développement d'une conduite nécessite la motivation, un but qui pousse à l'action. Thill

\footnotetext{
${ }^{16}$ Gimenez M., op. cit.

${ }^{17}$ Louart P., « Maslow, Herzberg et les théories du contenu motivationnel», Les Cahiers de la Recherche CLAREE, IAE-USTL, 2003.
}

constate que «les différentes théories de la motivation analysent chacune l'un ou l'autre aspect du système motivationnel humain tels la formation des buts, le déclenchement des conduites, le processus de régulation et l'interruption de conduites ». Ce constat amène l'auteur à soutenir que ce processus général peut être décomposé en plusieurs étapes: les influences personnelles et sociales, la fixation des buts, l'impact sur l'environnement, l'évaluation de l'action entreprise et les attentes. Cette dernière étape correspond à des croyances relatives aux possibilités d'accès aux buts et résultent elles-mêmes des processus d'évaluation des conduites propres et celles d'autrui. C'est certainement pour tenir compte de ces attentes que Louart ${ }^{19}$ avance que «la théorie des attentes part du principe que les individus font d'abord un choix parmi les résultats supposés de certaines activités. En se basant sur leur intuition ou leur expérience passée, ils évaluent pour eux la possibilité d'obtenir des résultats souhaités en ayant la conduite appropriée ». Les activités ici, sont les attributions et les fonctions des agents du système de sécurité et de justice ivoirien. Les résultats supposés de ces activités sont les attentes légitimes des victimes, des usagers et de la population entière. En se basant sur leur expérience passée ou celle des proches, la population évalue pour elle, les résultats de l'imposition de peines sévères pouvant et devant exprimer la désapprobation de la société à

\footnotetext{
18 Thill E., «La motivation. Une construction progressive », Hors-série, n 19 , Décembre 1997/ Janvier 1998.

${ }^{19}$ Louart P., «Maslow, Herzberg et les théories du contenu motivationnel», Les Cahiers de la Recherche CLAREE, IAE-USTL, 2003.
} 
l'égard du crime ${ }^{20}$. Il s'agit là d'un système qui assure la sécurité et la défense des individus, ainsi que la protection des biens. De ce qui précède, nous pouvons affirmer avec Louart ${ }^{21}$ que «l'action raisonnée suppose que pour expliquer un comportement, on doive tenir compte de l'attitude de son auteur, mais aussi de normes subjectives interférant avec ses choix ». L'attitude des agents du système de sécurité et de justice influencerait alors directement la représentation du système en général. Pour l'auteur, les agents «n'agissent donc pas uniquement pour obtenir des avantages personnels. Ils participent à l'action collective pour disposer d'un cercle permanent de reconnaissance qui partage leurs valeurs, rend constante leur image d'eux-mêmes et contribue à rendre commune leur définition et la réalité ».

Une étude d'un échantillon non représentatif, mais avec des données qualitatives $\mathrm{Okpo}^{22}$, montre que $38 \%$ des individus victimes d'infractions pénales portent plainte pour réclamer plus de sécurité des biens et des personnes; alors que $62 \%$ des victimes de ce même échantillon ne font pas de déclaration de l'incident aux autorités compétentes à régler les litiges entre les particuliers. Comment celles-ci règlent-elles l'incident subi? Quelles sont les motivations d'une telle attitude? Les modes de résolution des victimisations subies non portées

\footnotetext{
${ }^{20}$ Lachambre S., La théorie de la dénonciation: émergence et institutionnalisation en droit criminel, Thèse soumise à la faculté des études supérieures et postdoctorales dans le cadre des exigences du programme de doctorat en criminologie. Département de criminologie, Faculté des sciences sociales, Université d'Ottawa, 2011.

${ }^{21}$ Louart P., op. cit.

${ }^{22}$ Okpo N. A., « Les plaintes en matière criminelle à Abidjan », Revue Internationale de Criminologie
}

à la connaissance d'une autorité, selon cette étude, se résument d'une part, en laisser tomber sans que l'affaire ne soit résolue soit $80 \%$ des victimes qui ne déclarent pas l'incident, et d'autre part, en règlement à l'amiable $20 \%$ de ces victimes qui ne signalent pas l'infraction. Les facteurs motivationnels de cette attitude des victimes sont de trois ordres: le manque de temps, la croyance religieuse et surtout, le manque de confiance aux autorités de sécurité et de justice. Les attentes de celles-ci n'étant pas satisfaites, elles voient la protection des biens et des personnes par les agents de ces institutions comme une grande illusion. Car dans l'imaginaire de la population, lorsqu'un individu a subi une infraction, les agents des institutions de sécurité et de justice doivent retrouver l'infracteur et le punir. Ainsi, les victimes et même toute la population pourront sentir et avoir l'impression de bénéficier de plus de sécurité, de protection de la part des autorités compétentes. Cependant, cet espoir reste une vue de l'esprit. Or, comme le relève Malinowski, les institutions sociales sont les réponses collectives à des besoins humains fondamentaux. Et le besoin de sécurité des biens et des personnes, donc le besoin de quiétude est une composante de ces besoins fondamentaux.

Quelle est l'expérience des institutions de sécurité et de justice dont dispose la population abidjanaise? Quelle est l'image ou la représentation des institutions ivoiriennes retenue par la population? Qu'est-ce qui motive une telle représentation? Ou encore, existe-il une relation entre l'expérience vécue dans ces institutions et la perception que les abidjanais ont de celles-ci? Cette étude se propose

et de Police Technique et Scientifique, Vol. LXVIII, 
d'apporter quelques éléments de réponse à ce questionnement à travers trois objectifs.

Montrer les expériences vécues par les abidjanais dans les institutions de sécurité et de justice.

- Indiquer la perception des abidjanais de leurs institutions de sécurité et de justice

- Exposer la relation existant entre les expériences dont disposent les abidjanais et la perception de ceux-ci des institutions de sécurité et de justice.

Une hypothèse a conduit cette recherche. Les expériences d'insécurité au quotidien, d'insatisfaction d'assistance pour la protection des biens et des personnes des abidjanais expliquent l'image négative qu'ils retiennent de leur institutions de sécurité et de justice.

Les sujets de cette étude sont tous des résidents $\mathrm{du}$ district d'Abidjan, issus de toutes les catégories sociales, des deux sexes, et âgés de 20 ans et plus. Il s'agit de cent-sept (107) individus rencontrés dans divers lieux pour échanger sur leur perception et leur représentation des institutions de sécurité et de justice ivoiriennes. 39 personnes de sexe féminin et 68 de sexe masculin, tous de niveau d'études variables: deux non scolarisés, deux de niveau d'étude primaire, vingt-deux (22) de niveau secondaire et quatre-vingt-et-un (81) de niveau supérieur. Deux personnes sont sans emploi, quatre sont des planteurs, quarante-deux (42) étudiants, quatorze individus exerçant dans le secteur informel et quarante-un (41) autres sont des fonctionnaires et salariés du secteur privé.

Le recueil des données de cette étude a nécessité l'administration d'un questionnaire de vingt-et- une questions qui ont orienté les échanges et les entretiens avec les cent-sept (107) personnes rencontrées au cours de l'enquête. Au cours du mois de novembre 2015, certains étudiants et nous même, après exposé des objectifs et la compréhension des questions, sommes rendus dans les rues d'Abidjan, dans les domiciles, dans les administrations publiques et privées pour échanger avec la population. La volonté d'exprimer leur mécontentement (qu'ils expriment d'ailleurs à chaque occasion de dénonciation des victimisations, quand ils sont dans ces institutions) a fait accepter volontiers de participer à l'enquête. Il n'y a donc pas eu assez de difficulté au cours de l'administration du questionnaire et des entretiens, à part celles qui feignaient ne pas avoir le temps pour vaquer à leurs occupations quotidiennes. La méthode quantitative a permis de faire le traitement statistique des données recueillies et la méthode qualitative a servi à l'analyse qualitative des discours, des échanges afin de comprendre les différentes perceptions et de saisir les représentations qui paraissent les éléments observables de la perception. Il faut d'emblée signaler que les interventions des répondants se sont beaucoup appuyées sur la culture d'origine des uns et des autres. 


\section{Résultats.}

\begin{tabular}{|l|l|l|l|}
\hline \multirow{2}{*}{ Victimes } & \multicolumn{3}{|l|}{ Nombre de personnes } \\
\cline { 2 - 4 } & Femmes & Hommes & Total \\
\hline Oui & 33 & 48 & 81 \\
\hline Non & 6 & 20 & 26 \\
\hline Total & 39 & 68 & 107 \\
\hline
\end{tabular}

Tableau n¹: Victimisations des personnes recontreés

$\mathrm{Au}$ cours de l'enquête, nous avons rencontré cent sept (107) personnes dont trente-neuf (39) de sexe féminin et soixante-huit (68) de sexe masculin. Trente-trois (33) personnes de sexe féminin et quarante-huit(48) de sexe masculin ont été victimes d'au moins une infraction durant ces douze derniers mois. Six (6) personnes de sexe féminin et vingt (20) de sexe masculin n'ont pas été victimes. Au total, quatrevingt-et-une (81) personnes sur les cent sept (107), soit $76 \%$ de l'échantillon ont été victimes et vingt six (26) sur 107, soit $24 \%$ de l'échantillon n'ont pas été victimisés.

\begin{tabular}{|l|l|l|l|l|}
\hline \multirow{2}{*}{$\begin{array}{l}\text { Déclaration de } \\
\text { l'incident }\end{array}$} & \multicolumn{4}{l}{ Nombre de personnes } \\
\cline { 2 - 5 } & Féminin & Masculin & Total & $\%$ \\
\hline Oui & 12 & 22 & 34 & 42 \\
\hline Non & 21 & 26 & 47 & 58 \\
\hline Total & 33 & 48 & 81 & 100 \\
\hline
\end{tabular}

Tableau ${ }^{\circ} 2$ : Déclaration de l'incident

Des 81 victimes, 34 soit $42 \%$ ont fait la déclaration de l'incident subi et 47 soit $58 \%$ n'ont pas pu faire de déclaration à une quelconque autorité. Il y a 12 , soit $15 \%$ des personnes de sexe féminin et 22 , soit $27 \%$ des personnes de sexe masculin qui déclarent l'incident vécu. $58 \%$ des individus victimes de l'échantillon ne dénoncent pas leurs victimisations.

\begin{tabular}{|l|l|l|l|l|l|l|}
\hline \multirow{2}{*}{ Déclaration } & \multicolumn{4}{|l|}{ Nombre } & \multicolumn{3}{l|}{ Résolution } \\
\cline { 2 - 7 } & Féminin & Masculin & $\%$ & Oui & Non & Total \\
\hline Oui & 12 & 22 & 42 & 13 & 21 & 34 \\
\hline Non & 21 & 26 & 58 & 15 & 32 & 47 \\
\hline Total & 33 & 48 & 100 & 28 & 53 & 81 \\
\hline
\end{tabular}

Tableau $\mathbf{n}^{\circ} 3$ : Déclaration et résolution de l'incident

Pour les 34 personnes sur 81 de l'échantillon ayant déclaré l'incident à une autorité quelconque, leur sécurité et leur protection, ainsi que leur défense en cas d'attaque extérieure ou d'une agression sont les missions des institutions de sécurité et de justice. D'ailleurs, voici le résumé des échanges que nous avons eus avec elles, rendant compte de leur espoir de vivre une vie paisible en présence de ces institutions.

"J'ai déclaré l'incident pour que l'auteur soit arrêté et que justice soit rendue». "J'ai déclaré l'incident pour assurer ma sécurité et recevoir mon dû ». "J'ai déclaré lincident car je pense que les policiers peuvent le régler». "Si j'ai déclaré l'incident, c'est parce que cela me fait mal 
et je voudrais que la police me défende pour me faire justice ». "J'ai déclaré l'incident parce que depuis tout le temps que nous connaissons ce monsieur, il a la réputation d'être un grand voleur. Nous voudrions que la police nous aide à retrouver la quiétude, car c'est un véritable calvaire, une terreur que nous vivons avec lui ». "J'ai déclaré l'incident à la police parce que j'avais confiance en elle et surtout, c'est parce que les policiers sont tenus de me protéger et de me rendre justice en cas d'injustice subie ». "Je déclare l'incident en vue des poursuites judiciaires et avoir gain de cause ». "C'est pour punir le voleur et retrouver mes biens. Aussi, j'estime que la police est l'institution habilitée à rendre justice et à régler les éventuels litiges». "J'ai déclaré l'incident à la police et à la gendarmerie dans l'espoir d'avoir une suite favorable. Que les auteurs soient apprébendés et que justice soit faite ». "Je suis allé à la police déclarer l'infraction du fait de l'importance des pertes. C'est donc dans l'espoir que mon dû soit rendu ». "J'ai déclaré l'incident parce que j'ai été deux fois victime et je me suis toujours dit qu'une infraction vaut la peine d'être dénoncée malgré la réticence de certains proches qui disent ne pas faire confiance à la police ». "J'ai été gravement blessé et j'ai perdu beaucoup d'argent. Je me dis alors qu'en déclarant les agressions aux autorités compétentes, elles peuvent multiplier les actions de protection des biens et des personnes ».

Ainsi, pour de telles personnes qui croient en l'efficacité des institutions de sécurité et espèrent retrouver la tranquillité en leur présence, elles ne peuvent que leur rendre compte des différentes mésaventures subies.

Cependant, 13 personnes, soit $38 \%$ des répondants ayant déclaré l'incident et donc $16 \%$ des victimes ont pu avoir satisfaction. Alors que 21 , soit $62 \%$ de celles qui ont déclaré l'infraction correspondant à $26 \%$ des victimes disent être toujours dans l'attente et affirment alors que leur incident n'a pas été réglé. "Je suis toujours en attente. La police me fait savoir que l'enquête suit son cours 》".

15 victimes n'ayant pas déclaré leur incident avancent que leur litige a été réglé. La vengeance, le règlement à l'amiable et le pardon ou ne pas régler ou tout simplement laisser tomber sans règlement sont les trois modes de règlement qui nous ont été présentés. Trois personnes, notamment des commerçants ont usé de violences en mettant en place des groupes d'autodéfense. «L'incident a été réglé par la mise sur pied d'un groupe de jeunes vigiles pour les commercants afin d'exercer leurs activités dans la quiétude ».

Deux personnes dont une comptable et une personne exerçant dans le secteur informel affirment avoir pardonné. "J'ai pardonné et que son malheur ne parte pas de moi». Les dix autres ont réglé leur incident à l'amiable. "L'incident a été réglé de façon pacifique en partant voir le chef du fumoir de Yao sébi, sous quartier de Yopougon ». "L'incident a été réglé à l'amiable, par arrangement $»$.

$\mathrm{Au}$ total, des personnes qui ont déclaré l'incident, $16 \%$ des victimes ont pu trouver satisfaction alors que $26 \%$ ont été insatisfaits, quant au règlement de l'incident vécu. 18,5\% des victimes résolvent elles-mêmes leur litige soit par la vengeance, le règlement à l'amiable ou le pardon, soit en laissant tomber. 39,5 \% ne déclarent pas l'infraction et également ne prennent aucune initiative pour solutionner cette infraction subie. Les répondants sont au nombre de 47 , soit $58 \%$ des victimes ne déclarant pas l'incident.

$\mathrm{Au}$ regard des résultats en notre possession, nous pouvons affirmer que seulement 13 victimisations soit $16 \%$ ont été résolues par les institutions de sécurité et de justice. 68 
infractions, soit $84 \%$ n'ont pu être réglées soit pour leur non dénonciation, soit par la complexité du litige ou l'inefficacité des institutions ou encore leur non prise en compte par ces institutions compétentes.

En voici quelques témoignages des personnes rencontrées. "Je n'ai pas déclaré l'incident à cause des différentes expériences d'insatisfaction. Elles m'ont montré que ces affaires étaient toujours classées sans suite ». "Je n'ai pas déclaré l'incident car je pense que cela n'aboutira pas et pour éviter les longues démarches sans suite ». "Ma déclaration ne sert à rien puisqu’ils me demanderont de l'argent et ils ne pourront pas mener d'enquête. Je vois alors en ces démarches, une perte de temps ». "Je n'ai pas déclaré l'infraction. J'ai préféré garder le silence parce qu'à mon sens, il n'y aurait pas d'issue favorable. Je serais même gêné et découragé par les nombreuses lenteurs administratives injustifiées ». "Je n'ai pas fait de déclaration parce que j'étais dans l'obligation de ne pas le faire. Je pense même que cela n'apporterait pas de solution à mon problème ». "Je n'ai pas fait de déclaration de l'incident car j'estimais qu'il n'était pas aussi grave que cela, c'était juste un portable téléphone qui m'a été volé. L'objet m'importait peu par rapport à ma vie quelque soit son prix». "Je n'ai pas signalé l'incident car à mon avis, les institutions de sécurité et de justice sont corrompues et sont souvent inefficaces avec des procédures longues et interminables qui prennent suffisamment de temps et d'argent inutilement ». "Je n'ai pas dénoncé l'incident parce que généralement, lorsqu'on informe la police qu'on est victime d'infraction, il n'y a jamais de carburant pour aller sur les lieux». "Je n'ai pas dénoncé l'incident parce que les autorités policières n'interviennent pas pour les infractions comme l'agression. Elles me demanderont de mettre la main sur l'agresseur et de venir avec lui pour qu'elles commencent leurs longues procédures ».
Ces témoignages nous amènent à tenir compte des différentes expériences des uns et des autres dans les institutions chargées de la sécurité et de la défense des citoyens.

\section{Expériences vécues au sein des} institutions de sécurité et de justice.

Nous avons dénombré 69 personnes, soit 64,5 $\%$ de l'échantillon qui se sont rendues dans une institution de sécurité ou de justice pour l'insécurité vécue ou dont elles ont été des témoins ou subies par un proche. Trois grands groupes se dégagent de ces caractéristiques : 14 non victimes, proches d'une victime ou témoins d'insécurité. 21 victimes qui n'ont pas fait de déclaration mais qui se sont déjà rendues dans une institution de sécurité. 34 victimes qui ont eu à faire une dénonciation de leur victimisation. Pour les non victimes qui ont accompagné des proches ou qui sont allées dans une institution de sécurité ou de justice pour témoigner, l'accueil est bon pour 4 personnes. Moyennement bon pour 4 autres personnes et passable ou pas du tout satisfaisant ou même négligeant pour 6 personnes. "Je trouve l'accueil et le comportement bon puisque chacun a eu à expliquer de quoi il était question ». "L'accueil était bien parce que les agents ne m'ont pas brutalisé. Ils m'ont posé des questions qui m'ont permis de dire ma part de vérité sur les faits ». "L'accueil est négligeant et pas satisfaisant car lorsque nous sommes arrivés, en voyant la victime en pleurs avec les blessures, les agents nous ont même rejetés en nous demandant de prendre tout notre temps pour pleurer et après avoir vidé toute larme du corps, nous allons nous rendre dans un hôpital afin de soigner les blessures et seulement après revenir dans une institution pour porter plainte. Il faut souligner que tout ce discours 
a été tenu sous un air moqueur, dédaigneux au moment où l'agresseur nous narguait ».

Le jugement porté sur l'accueil et le comportement des agents des institutions de sécurité et/ou de justice par les victimes qui n’ont pas signalé leur victimisation, mais qui se sont déjà rendues dans une institution part de quelques fois courtois au comportement d'intimidation, de frustration, en passant par le désintéressement ou le mauvais accueil et comportement. "Je trouve que l'accueil des agents est un accueil de légèreté, de désintéressement. Ils ont un comportement d'intimidation qui m'a finalement frustré ». "Je trouve que l'accueil et le comportement des agents sont mawvais. Ils n'ont aucune considération pour l'homme. Ils pensent toujours à l'argent puisque l'idée de corruption prend toujours le dessus». "L'accueil n'est pas du tout chaleureux. Il n'est pas à la mesure de nos attentes, ils sont très mawvais ». "L'accueil et le comportement sont parfois courtois, mais pas toujours.

Les agents ne sont pas prompts lorsqu'ils sont sollicités ».

Le dernier groupe des personnes rencontrées qui ont porté un jugement sur l'accueil et le comportement des agents des institutions de sécurité et de justice est celui des victimes ayant déclaré leur infraction. Ce groupe peut être subdivisé en deux sous groupes; celui des personnes qualifiant l'accueil et le comportement de bon, de satisfaisant. Elles sont au nombre de 11 dont trois de sexe féminin et huit de sexe masculin. "L'accueil est bien et le comportement satisfaisant». "L'accueil est acceptable, bienséant. Certains sont exemplaires, d'autres non et le comportement est bien, courtois ». «Un bon accueil, mais le comportement n'est pas trop plaisant, cependant, acceptable tout de même ».

Le deuxième sous groupe est celui des personnes jugeant l'accueil de mauvais et le comportement hautin, agressif, peu courtois. Il est composé de 9 individus de sexe féminin et 23 de sexe masculin. "L'accueil est moyen et le comportement hautin, peu courtois». "L'accueil est froid et le comportement distant, agressif dans le verbe, peu agréable ». "Mon jugement est qu'à la police, c'est un véritable bordel, à la gendarmerie, c'est un peu bon. Le comportement est difficile à qualifier mais ce n'est pas encourageant». "Les plaignants ne sont pas toujours bien accueillis et ils relâchent le coupable dans bien des cas, malgré l'existence de preuves». "L'accueil est médiocre et le comportement anormal, victimisant, culpabilisant ». "L'accueil est un peu perplexe je l'avoue, pas trop rassurant. Pour le comportement, il faut dire que c'est apparemment la routine chez eux, ils ne sont pas trop pressés ». "Les agents ont un très mauvais accueil et un mauvais comportement». "Les agents ont un mawvais accueil. Le comportement est la tendance à rejeter la faute sur la victime ».

Au total, 8 personnes sur les 14 non victimes et 11 des victimes ayant fait une déclaration, soit $27,5 \%$ trouvent l'accueil et le comportement dans les institutions de sécurité et de justice bons, satisfaisants alors que $72,5 \%$ des personnes qui se sont rendues dans une institution de sécurité qualifient l'accueil et le comportement de mauvais, de frustrant et désagréables. A partir de ce qui précède, nous avons jugé opportun d'avoir une idée de la perception des abidjanais de ces institutions.

\section{Perception des abidjanais des institutions} de sécurité et de justice.

A l'interrogation de savoir si le répondant connait les attributions et les fonctions des agents de sécurité et de justice, 45 individus soit $42 \%$ disent ne pas connaître les attributions et fonctions des agents. 62 , soit $58 \%$ de 
l'échantillon affirment connaittre ces attributions et fonctions. A la suite de l'idée générale sur la connaissance des attributions et des fonctions des agents de sécurité et de justice, nous avons demandé au répondant d'apprécier le travail ou les services de ces agents. Trois différentes appréciations se dégagent des réponses données ; satisfaisant, peu satisfaisant et pas du tout satisfaisant. Le tableau suivant résume les réponses.

\begin{tabular}{|l|l|l|l|}
\hline $\begin{array}{l}\text { Appréciation du travail des } \\
\text { agents }\end{array}$ & Satisfaisant & Peu satisfaisant & Pas du tout satisfaisant \\
\hline $\begin{array}{l}\text { Non victimes ne s'étant } \\
\text { jamais rendues dans une } \\
\text { institution de sécurité ou } \\
\text { de justice }\end{array}$ & 3 & 4 & 5 \\
\hline $\begin{array}{l}\text { Non victimes s'étant } \\
\text { rendues dans une } \\
\text { institution de sécurité, } \\
\text { témoins déclarant et }\end{array}$ & 0 & 10 & 0 \\
\hline $\begin{array}{l}\text { Victimes non s'étant jamais rendues } \\
\text { ne une institution de } \\
\text { dans } \\
\text { sécurité }\end{array}$ & 20 & 6 \\
\hline $\begin{array}{l}\text { Victimes n'ayant pas fait } \\
\text { de déclaration }\end{array}$ & 7 & 7 & 7 \\
\hline $\begin{array}{l}\text { Victimes ayant fait une } \\
\text { dénonciation }\end{array}$ & 4 & 22 & 8 \\
\hline Total & 18 & 63 & 26 \\
\hline Pourcentage & 17 & 59 & 24 \\
\hline
\end{tabular}

Tableau $\mathbf{n}^{\circ} 4$ : Appréciation du travail des agents

18 personnes soit $17 \%$ de l'échantillon trouvent le travail des agents des institutions de sécurité et de justice satisfaisant. 63, soit $59 \%$ l'apprécient de peu satisfaisant et 26 , soit $24 \%$ de l'échantillon le déprécient en le qualifiant de pas du tout satisfaisant.

Pourquoi de telles appréciations? Pour comprendre et suivre la logique de leur jugement, nous avons reparti les répondants en cinq groupes.

Le groupe des non victimes qui ne se sont jamais rendues dans une institution de sécurité ou de justice, trios apprécient positivement le travail des agents. "Le travail des agents est satisfaisant parce qu'ils font leur boulot malgré tout».

Pour ceux du même groupe qui pensent que le travail des agents est peu satisfaisant ressortent les facteurs tels que trop de rackets, trop de corruptions, la lenteur du service, les agents ont les mains liées, ils ne sont jamais libres pour recevoir. "Je suis peu satisfait de leur travail parce que dans cette corporation, il y a trop de corruption ».

Pour le groupe des non victimes qui ont soit accompagné un parent, soit sont témoins, 4 personnes trouvent le service satisfaisant et dix le qualifient de peu satisfaisant. «Je suis satisfait du travail des agents. Cependant, le service est lent et on a l'impression que les agents ne sont jamais libres pour recevoir les usagers ». "Je suis peu satisfait du travail des agents. Une telle appréciation parce que ceux-ci focalisent leur attention sur la connaissance et les financiers ». "Je suis peu satisfait du travail des agents parce qu'ils ne répondent pas à mon attente. Une telle appréciation se justifie par l'appât du gain facile et la lenteur dans 
l'exécution de leur boulot ». "Je suis peu satisfait car très souvent, c'est celui qui porte plainte qui a raison ».

Pour les victimes n'ayant pas fait de déclaration et ne s'étant jamais rendues dans une institution de sécurité ou de justice au nombre de 26, elles ont appris des dires des amis ou de proches que le travail des agents est soit peu satisfaisant, soit pas du tout satisfaisant. "On entend autour de nous dire qu'il y a trop de légèreté dans leur comportement. C'est pourquoi je dis je suis peu satisfait ». "Je suis peu satisfait de leur travail parce que les agents de sécurité n'cuvrent plus pour le bonheur et la quiétude de la population ». "Je suis peu satisfaite du travail des agents car ils sont trop corrompus et ils reçoivent mal les usagers ». "Je suis peu satisfaite parce que les agents font ce qu'ils peuvent avec le peu de moyens dont ils disposent ». "Je suis peu satisfaite à cause du témoignage qu'on reçoit qui ne fait pas leur éloge. Ils ne mènent pas les enquêtes comme il se doit». "Je suis peu satisfait parce que nous sommes dans un pays de non droit où sévit la corruption et beaucoup d'affaires restent sans solution pour l'ensemble de la population. Les institutions pour moi, n'ont pas les pleins pouvoirs et sont souvent corrompues ». "Je ne suis pas du tout satisfait du travail des agents parce qu'ils ignorent la règle 'nul n'est audessus de la loi'. Cette règle n'est pas respectée et chacun s'attribue des pouvoirs à son gré». "Je ne suis pas du tout satisfait parce que dans l'ensemble, nous subissons toujours les mauvais coups parfois même de ceux sur qui nous comptons pour notre sécurité ».

Pour les victimes n'ayant pas fait de déclaration, mais qui se sont déjà rendues dans une institution de sécurité ou de justice, au nombre de 21, sept sont satisfaites, sept autres sont peu satisfaites et enfin, sept ne sont pas du tout satisfaites. "Je suis satisfait du travail des agents parce que le plus souvent, excepté l'accueil, j'ai la satisfaction. Ceux-ci jouent leurs rôles de loyauté et de partialité. Ils nous aident sur certains plans. Cependant beaucoup reste à revoir ». "Je suis peu satisfaite du travail des agents car ils amènent l'individu ou le plaignant à leur fournir toutes les informations et ils ne font pas d'enquête ». "Je suis peu satisfait du travail des agents parce que cela fait plus de trois ans ceux-ci n'arrivent pas à démanteler le réseau des agresseurs en bordure des voies du quartier Yao sébi non loin du 16 eme arrondissement de police de Yopougon. Ceci me laisse voir l'insuffisance et l'inefficacité de leur travail ». "Je ne suis pas du tout satisfait du travail des agents parce qu'ils n'ont aucun respect des usagers alors qu'ils veulent le respect et ils abusent de leur autorité. Ils ne répondent pas à temps quand on a besoin d'eux et ils sont corrompus $\gg$.

Pour les victimes ayant déclaré l'infraction subie, au nombre de trente-quatre, 4 sont satisfaites, 22 sont peu satisfaites et huit pas du tout satisfaites. "Je suis satisfait, seulement que le service est lent et les agents ne sont jamais libres. Ils ont les moyens de lutte contre les actes délictueux et ils sont disponibles pour la population". "Je suis peu satisfait parce qu'on ne voit pas de réels résultats. Ils nous répondent toujours, l'enquête suit son cours. Voilà ma seconde infraction, le coupable n'a pas pu être identifié ni arrêté ». "Je suis peu satisfait parce que les agents sont incompétents et corrompus. Ils préferent se laisser corrompre la plupart $d u$ temps par les criminels. Ils ne tranchent pas véritablement les litiges et sont au pas de ceux qui sont fortunés. Souvent le plaignant et le mis en cause sont dans l'obligation d'une entente pour un règlement à l'amiable ». "Je suis peu satisfait parce que je pense qu'il y a absence de conscience professionnelle de leur part. Il y a abus de pouvoir et corruption ». "Je suis peu satisfait à cause de la lenteur et du non aboutissement des enquêtes. Ce qui peut s'expliquer par une formation insuffisante et de peu de moyens pour satisfaire la population ». "Je suis peu satisfait parce que les agents de police que j'ai rencontré n'ont pas écouté mes préoccupations. Ils sont 
parfois négligents et corrompus. Les soucis portés à leur connaissance restent sans solution concrète ». "Je ne suis pas du tout satisfaite du travail des agents parce que pour régler un problème, souvent le plaignant dépense suffisamment d'argent et puis vers la fin, ce n'est pas trop ça. Ce dernier n'obtient pas gain de cause. Aussi, ceux-là même qui sont chargés d'appliquer la justice, font subir l'injustice au sein même de l'institution de sécurité et/ou de justice. Bien qu'autorités compétentes, elles ne résolvent jamais les soucis des usagers qui les sollicitent ».

Dans le même ordre d'idée, nous avons demandé aux personnes de l'échantillon de dire si oui ou non les agents répondent favorablement lorsque leurs services sont sollicités.

Selon 34 personnes, soit $32 \%$ de l'échantillon, lorsque les services de sécurité sont sollicités, les agents répondent favorablement. Quant à 73 personnes, soit $68 \%$ de l'échantillon, les agents ne répondent pas favorablement. Cette réponse négative que donnent les agents aux sollicitations de la population est un indicateur de l'appréciation générale des services des agents des institutions de sécurité et de justice. En effet, l'interrogation qui cherchait à connaitre l'image retenue par le répondant du travail des agents donne les indications suivantes : 16 personnes, soit $15 \%$ gardent une image pas du tout bonne du travail des agents. 72 répondants, soit $67 \%$ de l'échantillon retiennent une image peu bonne et 19 personnes soit $18 \%$ intériorisent une bonne image du travail des agents de sécurité et de justice.

Quelles explications les individus donnent-ils dans chaque série d'image retenue?

Pour les $18 \%$ qui gardent une bonne image du travail des agents, les justificatifs peuvent se résumer aux propos tels, «je retiens une bonne image du travail de ces agents parce qu'ils veillent sur notre sécurité et celle de nos biens et c'est vraiment pas facile ». "Ils font leur travail, mais parfois ils ont beaucoup de cas à traiter. C'est ce qui fait que le service est lent et donne l'impression qu'ils ne font souvent pas leur travail ». "Malgré la situation, économique et financière du pays, ces agents donnent le meilleur d'eux-mêmes pour satisfaire la population ». "Je retiens une image bonne et non pas très bonne à cause quelques déceptions. Ces agents sont peu disponibles, le service est lent et il existe la corruption en leur sein ». "Pour moi planteur, les agents réagissent aux préoccupations de la population »’. Pour la grande majorité, $67 \%$ qui retient une image peu bonne soutient que «le travail ne se fait pas comme il se devait. Les agents ne font pas correctement leur travail, puisqu'en tant qu'agents de loi, ils devaient s'impliquer à fond et il y a trop de racket». "Ils attendent toujours quelque chose de nous avant de faire leur travail pour lequel la nation les emploie et les paie ». "Je retiens une image peu bonne parce que les agents répondent difficilement et tardivement aux sollicitations. Leur rendement est donc insuffisant. Ils ne sont pas réguliers sur le terrain. On les voit par coup d'éclat lorsqu'ils veulent montrer leur existence dans les moments où la grande criminalité est observable un peu partout». "Cette image est peu bonne pour ne pas dire pas bonne, car on a l'impression que les agents travaillent par contraintes. Ils ne rassurent pas vraiment la population, le travail est fait avec beaucoup de légèreté. Les seuls moments où ils donnent l'impression de travailler, c'est quand il y a des dessous de tables. Vous voyez, moi un sans emploi, que vais-je chercher à la police ou à la gendarmerie? J'ai été victime et je ne suis pas allé dénoncer l'infraction et je n'irai jamais là-bas. D'ailleurs, j'ai préféré garder le silence par simple gêne. J'ai laissé tomber, je préfere rester chez. moi que d'aller m'bumilier». "L'image est peu bonne il faut le dire. Aucun effort dans la résolution des litiges et autres 
infractions. Il y a d'abord un mawais accueil. Ensuite, leur comportement de corrompus, de racketteurs font qu'ils sont inefficaces. Enfin, je me dis qu'ils sont mal formés et n'ont pas la qualification requise pour ce travail. Je dirais une image pas du tout bonne, mais quelques rares agents émergent du lot, c'est pourquoi je dis peu bonne ». "Je garde une image peu bonne du travail des agents parce qu'ils s'intéressent peu aux demandes, aux sollicitations de la population. On a l'impression qu'ils ne sont pas intéressés par leur boulot. Pour moi, ils ont reçu une mawvaise formation et ils font une mawaise utilisation du matériel de sécurité disponible ». "Je retiens une image peu bonne du travail des agents de sécurité parce qu'il y a trop de magonilles, de lenteur pour favoriser la magouille, et ces agents sont en général peu attentifs aux préoccupations des populations. Ils abusent leur autorité". "Je retiens une image peu bonne du travail des agents de sécurité. En tant que activiste des droits de l'homme, apprendre ou être témoins des actes tels que les agents demandent indirectement des pots de vin pour suivre certains dossiers, cela est éccurant. On se dit qu'ily a absence de conscience professionnelle ». "J'ai une image peu bonne parce que j'attends beaucoup plus d'eux. Qu'ils soient plus prompts, persévérants et efficaces. Que le service ne soit pas payant, qu'ils arrêtent la corruption. La lenteur fait que les résultats sont peu satisfaisants. Aussi, beaucoup font la politique maintenant. Il faut connaître quelqu'un au sein des institutions, si non, l'affaire trainera pendant longtemps. L'argent à tout gâté et les agents ne sont pas motivés à défendre la cause de la population ».

Pour ceux qui ont gardé une image pas du tout bonne, ils retiennent également la lenteur du service, la corruption, l'abus du pouvoir d'autorité compétente, l'insouciance de la gravité de l'incident et de l'absence de la conscience professionnelle, des agents qui ne s'investissent pas sérieusement dans leur travail.
Cette image négative du travail des agents des institutions de sécurité et ou de justice se répercute sur l'image du système de sécurité et de justice en général. Car l'interrogation qui cherchait à connaître le lien entre l'image que le répondant retient du travail des agents; les expériences au sein des institutions, les réponses négatives aux sollicitations, et sa perception de ce travail et du système en général, donne des indications suivantes : 17 personnes, soit $16 \%$ garde une image pas du tout bonne du système. 66 soit $62 \%$ retiennent une image peu bonne du système. Seulement 22 , soit $20 \%$ pensent que le système est bon et $2 \%$ le trouvent très bon. Au total, $78 \%$ de l'échantillon n'ont pas une bonne image du système de sécurité et de justice ivoirien et $22 \%$ disent avoir une bonne image du système.

Pour l'homme d'affaires, de niveau secondaire, "l'image est très bonne parce que le système permet d'éviter des inconduites, des déviations sociales ». Un comptable de niveau supérieur qui retient une bonne image du système, a tout de même un avis mitigé puisque selon lui, "l'image est bonne pour la sécurité et moins bonne pour la justice où il faut avoir des moyens financiers pour aller au terme de son affaire en justice ». L'activiste des droits de l'homme de niveau supérieur garde une bonne image du système puisqu'il avance que "le système en luimême est bon. Cependant, les agents ne travaillent pas selon les règles de déontologie ».

$78 \%$ de l'échantillon déprécient le système en affirmant qu'il n'a pas une bonne audience auprès de la population et cette image négative ressort les facteurs comme le favoritisme, la corruption, l'achat des services publics, la partialité, la faiblesse du système qui est à la solde des politiques et des hommes nantis etc. 
Un opérateur économique trouvant le système négatif avance "notre système avait déjà du plomb dans l'aile avant 2010, mais maintenant, elle n'existe que de nom ». Un étudiant retenant une mauvaise image du système accuse. «Le système n'est pas bon à cause de l'indisponibilité des agents qui l'animent et cela n'encourage pas la population à recourir à ses services. L'accueil et le comportement des agents sont responsables de l'image négative du système de sécurité et de justice ». Un autre étudiant et une commerçante ajoutent. "Notre justice a les mains liées. La police travaillant dans des conditions difficiles avec peu de moyens, les agents font payer les services publics à la population. Les gouvernants ne font pas suffisamment pour les jeunes et le système est beancoup basé sur la répression plutôt que la prévention qui devrait se baser sur une politique globale de sécurité ». Pour un planteur, un opticien et un enseignant du supérieur, "les lois sont votées, mais très peu sont appliquées sur le terrain à cause de la corruption et du manque de responsabilité des agents. Autrement, le système réagit aux préoccupations de la population par le vote des lois, mais hélas».

Lorsque nous avons demandé au répondant d'établir un lien entre sa manière de voir le système et ses agents et sa représentation générale du système, les différentes expériences vécues dans ces institutions ont orienté les réponses des personnes rencontrées. 86 personnes, soit $80 \%$ donnent une réponse affirmative alors que 21 , soit $20 \%$ disent que les différentes expériences dans ces institutions n'ont pas de lien avec leur représentation du système. Qu'est ce qui modifierait la perception actuelle du système et de ses agents? Les réponses sont aussi diverses que les griefs formulés à l'endroit des agents et du système.

Existe-il des voies de recours en cas d'insatisfaction des usagers du système de sécurité et de justice? La réponse à une telle préoccupation est donnée par deux personnes que nous avons sollicitées dans le cadre de cette étude. Un lieutenant de police qui a connaissance des convocations des agents à l'inspection générale de la police et un enseignant-chercheur de l'université qui enseigne les sciences juridiques.

La réponse donnée à cette interrogation par ces deux répondants est sans ambigüité. Il existe bel et bien des voies de recours des usagers ou des justiciables insatisfaits, qu'il s'agisse des agents de la police, de la gendarmerie et ou de la justice. Lorsqu'un citoyen ivoirien est insatisfait de l'accueil, du comportement d'un agent et de l'issue donnée à sa préoccupation, à son litige, celui-ci peut d'abord chercher à régler l'affaire au niveau du commissariat de police ou de la brigade où le litige a été porté. Le responsable du commissariat, c'est-à-dire le commissaire de police ou le commandant de brigade convoque l'agent en cause et trouve une solution; la satisfaction de l'usager. Pour la reconnaissance de l'agent fautif, il existe des séances nommées « revue visage » à la compagnie républicaine de sécurité (CRS) et dans les commissariats. Dans le cas où l'usager se rend directement à l'inspection ou au commandement supérieur, le l'affaire est ramenée au niveau du commissariat ou de la brigade. Autrement, le commissaire ou le commandant de brigade est appelé pour donner une suite à la préoccupation de l'usager. Si le règlement au niveau du commissariat ou de la brigade ne satisfait toujours pas l'usager, celui-ci peut porter l'affaire à un niveau supérieur qui est l'inspection générale du travail basée à la préfecture de police ou au commandement supérieur ou au tribunal militaire pour $\mathrm{y}$ 
convoquer l'agent en question. Cependant, il faut des arguments solides, des preuves suffisantes à la personne plaignante pour incriminer l'agent. Très souvent, fait remarquer le lieutenant, les affaires ou litiges que les usagers portent à la connaissance de l'inspection du travail sont les abus de confiance, les escroqueries pour lesquels, au premier niveau, les responsables contraignent l'agent au remboursement intégral de la somme escroquée ou abusée. Au niveau de la justice, lorsqu'un citoyen est insatisfait des agissement des agents et du jugement de son affaire, il fait tout simplement appel de la décision à un niveau supérieur au tribunal de première instance.

La population et partant, les ivoiriens ont-ils connaissance de l'existence de ces structures pour demander réparation ou une amélioration des services des institutions ? Nous sommes tentés de répondre par la négative. Dans tous les cas, pour la grande majorité, puisque beaucoup se résignent en ne signalant pas les incidents, se disant qu'ils n'auront pas gain de cause. Pour ceux qui y partent, les griefs ne sont pas pris en compte, du moins, il leur ait demandé de donner des raisons ou preuves solides et suffisamment consistantes pour incriminer l'agent supposé fautif. Qu'est ce qui modifierait selon le répondant, la perception actuelle du système et de ses agents? Les différentes réponses sont résumées dans les propositions suivantes.

Les propositions pour un système fréquentable, conçu pour la population, prenant en compte les préoccupations des ivoiriens et ivoiriennes devra réorienter la politique criminelle globale. Elle devra commencer par la formation des agents de sécurité et de justice à bas âge dans les écoles militaires. C'est alors après une telle formation et seulement après elle que les agents doivent être repartis dans les différents services du système. On y inculquera aux apprenants, l'esprit civique et citoyen. Ces apprenants une fois sur le terrain devront se considérer comme étant en mission aux services des populations. Ainsi, personne ne se croira ou ne se mettra au-dessus de la loi et ne s'arrogera ou n'abusera des pouvoirs d'autorité à elle conférés. Dans ces conditions, nous aurons un système qui ne va pas à la dérive. Il y aura une réduction considérable ou une disparition totale du racket, un changement radical du comportement des agents et du système. On observera certainement l'arrêt de la corruption, de la partialité et un peu plus d'efforts pour le respect des lois, des citoyens et de leurs droits. Et comme l'entrée dans cette école militaire sera volontaire et sans contrainte, mais avec conviction, nous aurons l'amour du travail, la rigueur, l'engagement dans le travail, la disponibilité et l'honnêteté des agents dans le travail. Ceux-ci assisteront les personnes en danger et celles qui sollicitent leurs services. Pour un tel travail, il faudra revoir la grille salariale des agents qui devront se débarrasser du manteau de quelqu'un qui est en perpétuelle quête d'argent.

Après un tel résumé des propositions des personnes rencontrées, nous retranscrivons à présent les propositions des répondants dans les termes qu'ils ont utilisés. «Ce qui modifierait ma perception actuelle du système et de ses agents est la suppression des brebis galeuses, la restructuration $d u$ système afin de mettre des hommes et des femmes bonnêtes, loyaux, garants de la justice ». "Il faut que les agents aient l'amour de leur travail. Qu'ils soient disponibles quelque soit la personne qui se présente à eux. Car, la plupart du temps, ceux-ci s'occupent plus des affaires juteuses pour eux ». "La modification actuelle de 
ma perception du système et de ses agents nécessite le respect des civils, l'application des lois, plus de conscience professionnelle de la part des agents, l'impartialité et l'incorruptibilité des agents ». "Que l'amour de la patrie leur soit inculqué et que la politique ne rentre pas dans les affaires de police et de justice. Il faut donc l'impartialité totale du système par rapport au régime politique ». «Pour modifier la perception actuelle que la population a du système de sécurité et de justice et de ses agents, il faut former les agents de sécurité et de justice à bas âge comme à l'EMPT' de Bingerville. Car plusieurs entrent dans ces corps de métiers sans conviction, juste pour avoir un salaire mensuel et espérer travailler jusqu'à la retraite. Il faut alors l'amour et la volonté de chaque agent à l'exercer». "Pour percevoir le système et ses agents autrement, il faudrait que ceux-ci changent leurs manières de travailler, notamment dans l'approche, le traitement des cas qui leur sont soumis ou présentés. C'est pourquoi il faut une éducation des agents du système concernant l'accueil des victimes, des usagers, sur la bonne utilisation des équipements de travail. Surtout, ne pas utiliser les fonds à la disposition du système en général à des activités de prestige, mais plutôt à des fins de réduction de la criminalité". "Le système en général et ses agents en particulier gagneraient à être plus droits et à rechercher la vérité dans le traitement des litiges en faisant valoir le respect des droits de l'homme". "Mon opinion et ma perception changeront si les agents changent, s'ils s'améliorent. Ils doivent avoir un esprit civique et citoyen, revêtir le manteau de missionnaires intrépides au service de leur serment".

\section{Discussion et conclusion.}

L'étude menée sur 107 personnes à Abidjan, cherche à connaître la perception et la représentation induite des institutions de sécurité

\footnotetext{
${ }^{1}$ Ecole Militaire Préparatoire Technique.
}

et de justice ivoiriennes par la population abidjanaise. Les résultats de cette étude montrent que les différentes expériences d'insécurité vécue au quotidien, les insatisfactions quant aux sollicitations des services des institutions de sécurité et de justice, ainsi qu'à la protection pas du tout satisfaisante des personnes et de leurs biens par les agents, le mauvais accueil, le comportement de négligence et de frustration etc., laissent une image négative de ces institutions et de ses agents dans la conscience de la population. Au total, $83 \%$ des personnes interrogées ont une appréciation négative du travail des agents de ces institutions qu'elles qualifient de pas satisfaisant. Cette insatisfaction par rapport au travail des agents a une répercussion sur l'image du système en général. Statistique canada (2011) avait également mis en exergue l'insatisfaction des Canadiens qui ont été plus sévères à apprécier le travail des institutions de sécurité. Cette étude expose que «dans l'ensemble, les résidents ont été sévères en ce qui concerne les efforts de la police pour informer le public en matière de prévention de la criminalité, pour traiter les gens de façon équitable et pour répondre rapidement aux appels. Les résidents de trois territoires étudiés au canada sont plus sévères à l'égard du système judiciaire et du système correctionnel que la police. Les personnes qui avaient eu affaire aux tribunaux étaient plus susceptibles de déclarer que les tribunaux font un mauvais travail pour ce qui est de rendre justice rapidement et d'aider les victimes ». $78 \%$ de l'échantillon de cette étude retiennent une image peu bonne du système.

La population abidjanaise déprécie le système dont elle garde une image négative en affirmant qu'il n'a pas une bonne audience à cause du 
favoritisme, de la corruption, de l'achat des services publics, de la partialité, de la faiblesse du système à la solde des politiques et des hommes nantis. En fin de compte, 80 \% soutiennent que les différentes expériences dans ces institutions ont un lien avec leur représentation négative du système et de ses agents. Dans l'ensemble, les abidjanais se sentent en sécurité et ne craignent pas pour leur sécurité personnelle en présence des forces de l'ordre. C'est alors la proximité qui est demandée à la police, à la gendarmerie et aux autres forces pour la protection et la défense des biens et des personnes. Les griefs reprochés aux institutions de sécurité et de justice ont alors pour conséquences le non signalement des incidents de victimisation à la police et à la gendarmerie soit $58 \%$. Une proportion semblable a été observée $\mathrm{Okpo}^{2}$ où $62 \%$ ne dénoncent pas l'incident et $34 \%$ seulement des victimes déclarent les victimisations. C'est également le cas de Perreault et Brennan ${ }^{3}$, exploitant l'ESG 2009, exposent que $30 \%$ des victimes d'un crime dans les territoires comme dans les provinces ont signalé les incidents aux services de police. Nos objectifs sont de rendre compte des expériences vécues par les abidjanais dans les institutions de sécurité et de justice, d'indiquer leur perception et de relever l'existence ou non d'une relation entre les expériences dont ils disposent et la représentation de ces institutions. Des résultats en notre possession, nous pouvons affirmer que nos objectifs sont atteints et que notre

\footnotetext{
${ }^{2}$ Okpo N. A., «Les plaintes en matière criminelle à Abidjan », Revue Internationale de Criminologie et de Police Technique et Scientifique, Vol. LXVIII, 2015.

${ }^{3}$ Perreault S., Brennan, S., La victimisation au Canada, 2009, Centre Canadien de la statistique juridique, Satatistics Canada-N85-002X, 2010.
}

hypothèse est confirmée d'autant plus que l'image négative retenue des institutions de sécurité et de justice est motivée par les expériences d'insécurité vécues au quotidien, les insatisfactions d'assistance pour la protection et la défense des biens et des personnes par les agents de ces institutions. L’image négative retenue du système s'est installée progressivement, au fil du temps dans la conscience collective des abidjanais qui avaient une certaine appréhension des hommes en tenue au cours de la période où exercer dans ces corps de métiers faisait la fierté de tout un peuple. C'est pourquoi il est impérieux que les services des institutions de sécurité et de justice mettent à jour leur fonctionnement et que les services rendus par ces institutions fassent l'objet d'une évaluation par des représentants extérieurs et non-institutionnels en toute transparence avec la population afin de ne pas rester déconnectées des attentes des citoyens.

\section{Bibliographie.}

- Algava E., Bèque M., « Perception et vécu des comportements intolérants. Une analyse $\mathrm{du}$ modèle "Relation aux autres" de l'enquête Histoire de vie", Economie et statistique, volume 393, $\mathrm{n}^{\circ} 1,2006$, pp 115 150.

- Aucoin K., Beauchamp D., Répercussion et conséquences de la victimisation, ESG 2004. Statistique Canada-n ${ }^{\circ} 85-002-X I F$ au catalogue, vol. 27, n¹, 2008, Ottawa, Centre Canadien de Statistique Juridique.

- Bansept C., Prévention de la délinquance, mobilisation civile et recherche de la juste peine. Sécurité publique et relations police-population: passer d'une police d'ordre à une police au service du citoyen. Rapport de mission du conseil national des villes à Montréal du 28 septembre au 3 octobre 2008, Montréal, 2013.

- Brodeur J-P., Les visages de la police : pratiques et perceptions, 2003. Document produit en 
version numérique, site web : http://classiques.uqac.ca/

- Carrasco V., Chaussebourg L., Creusat J., «Les déterminants du dépôt de plainte : le type d'agression subie devance de loin les caractéristiques de la victime ", Economie et statistique, n॰448-449, 2011.

- Chaussebourg L., Se déclarer victime: de l'atteinte subie au dépôt de plainte, Infostat Justice, avril 2010, $\mathrm{n}^{\circ} 109$, Ministère de la justice et des libertés, France.

- Chaussebourg L., Se déclarer victime: de l'atteinte subie au dépôt de plainte, Bulletin d'information statistique, Infostat Justice, 2010, n¹10, Ministère de la justice et des libertés, France.

- Fassin D., The embodiment of inequality, 2003, Document produit en version numérique, site web : http://www.ncbi.nlm.nih.gov/pmc/articles /PMC1326443/

- Gimenez M., La psychologie de la perception, Flamarion, 1997.

- Hill K. J., Réaction des victimes au traumatisme et conséquences sur les interventions: étude et synthèse de la documentation, Ministère de la Justice, Canada, 2003.

- Hill K. J., Working with victims of crime: $A$ manual applying research to clinical practice, Department of Justice, Canada, 2003.

- Killias M., Staubli S., Biberstein L., Bänziga M., Iadanza S., Sondage au sujet des expériences et opinions sur la criminalité en Suisse. Analyse dans le cadre sondage national de victimisation 2011, Institut de criminologie, Université Zürich, 2011.

- Lachambre S., La théorie de la dénonciation: émergence et institutionnalisation en droit criminel, Thèse soumise à la faculté des études supérieures et postdoctorales dans le cadre des exigences du programme de doctorat en criminologie. Département de criminologie, Faculté des sciences sociales, Université d'Ottawa, 2011.

- Louart P., «Maslow, Herzberg et les théories du contenu motivationnel », Les Cabiers de la Recherche CLAREE, IAE-USTL, 2003.

- Okpo N. A., «Les plaintes en matière criminelle à Abidjan », Revue Internationale de Criminologie et de Police Technique et Scientifique, Vol. LXVIII, 2015.

- Perreault S., Brennan, S., La victimisation au Canada, 2009, Centre Canadien de la statistique juridique, Satatistics CanadaNo85-002X, 2010.

- Perreault S., Brennan, S., Criminal Victimization in Canada, 2009, Centre Canadien de la statistique juridique. SatatistiqueCanada-N85-002X, 2010.

- Richard E., Pacaud M-C, Perception du travail policier: le point de vue d'étudiants en techniques policières, Rapport PREP, 2008. Format électronique sur le serveur WEB du CDC. URL=http:/ /www.cdc.qc.ca/prep/786758richard-pacaud-perceptions-travail-policiercampusNDF-2008.PDF

- Sidebottom A., «On the correlates of Reporting Assault to the Police in Malawi », The British Journal of Criminology, vol. 55, n. 2, 2015, pp. 381-398.

- Sillamy N., Dictionnaire de la psychologie, Larousse, Evreux, 1976.

- Thill E., «La motivation. Une construction progressive », Hors-série, n¹9, Décembre 1997 / Janvier 1998.

- Tremblay R., Vers une écologie humaine, 1990. Document produit en version numérique, site web : http://classiques.uqac.ca/

\section{Annexe.}

Réflexion sur la perception des institutions de sécurité et de justice par les abidjanais. Echange avec la population abidjanaise dans le cadre de cette réflexion.

\section{Identification de l'enquêté}

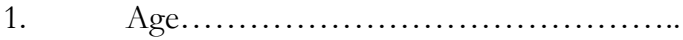

2. Sexe

3. Niveau d'étude.

4. Profession ou activité

Questions des échanges avec l'enquêté

5. Avez-vous été victime d'une infraction?

Oui..................Non.................

6. Si oui, avez-vous porté l'incident à la connaissance de :

la police, Oui..........Non...........

la gendarmerie, Oui.........Non...........

d'une autre autorité ? (à

préciser $). . . \ldots \ldots \ldots \ldots$................Non........

7. Si non, pourquoi n’avez-vous pas déclaré l'incident?

8. Si oui, pourquoi avez-vous déclaré l'incident? 
9. Si oui, l’incident a-t-il été réglé ? Oui............. Non............

10. Si non, comment l'incident a-t-il été réglé ? Vous est-il arrivé de vous rendre à la police, à la gendarmerie ou la justice pour un incident vécu? Oui.... Non

11. Si oui, comment trouvez-vous l'accueil et le comportement des agents?

Accueil....

Comportement.

12. Connaissez-vous les attributions et les fonctions de ces agents ? Oui......... Non.........

13. Comment trouvez-vous le travail ou les services des agents de ces institutions de sécurité et de justice?

Très satisfaisant........Satisfaisant.....Peu satisfaisant......Pas du tout satisfaisant..........

14. Pourquoi une telle qualification?

15. Ces agents répondent-ils favorablement lorsque vous sollicitez leurs services?

Oui................Non.
16. Quelle image retenez-vous du travail de ces agents?

Très bonne........Bonne......Peu bonne......Pas du tout bonne.........

17. Pourquoi une telle image?

18. Quelle image retenez-vous du système de sécurité et de justice en général ?

Très bonne........Bonne......Peu bonne......Pas du tout bonne..........

19. Pourquoi une telle image de ce système?

20. Votre manière de voir le système et ses agents a-t-il un lien avec vos différentes expériences? Oui...........Non..........

21. Qu'est ce qui modifierait votre perception actuelle du système et de ses agents?

22. Vous sentez-vous en sécurité en présence de ces institutions ou de ses agents dans votre vie de tous les jours?

Oui ........... Non. 